\title{
Do sub-mm sources and quasars form an evolutionary sequence?
}

\author{
O. Almaini \\ Institute for Astronomy, Royal Observatory, Blackford Hill, Edinburgh EH9 3HJ, UK
}

Received date will be inserted by the editor; accepted date will be inserted by the editor

\begin{abstract}
The high redshift sub-mm sources discovered by SCUBA are widely believed to represent the dust-enshrouded formation of massive elliptical galaxies. Given the strong evidence for a link between the formation of the spheroid and the growth of the central black hole, one might expect to see a luminous quasar at the nucleus of every SCUBA source. Somewhat surprisingly, however, only a very small fraction $(\sim 5 \%)$ are detected by Chandra with quasar luminosities. In this paper I discuss some of the implications of these results and discuss the accumulating evidence that sub-mm sources and quasars may represent different stages in the evolutionary sequence of a massive proto-spheroid.
\end{abstract}

Key words: quasars:general — galaxies:formation — galaxies:evolution — gravitational lensing — X-rays

\section{Introduction}

In recent years it has become clear that essentially every massive galaxy hosts a supermassive black hole (Kormendy \& Richstone 1995). In particular, the remarkable relationship between the black hole mass and the spheroidal velocity dispersion suggests a strong link between an early epoch of quasar activity and the formation of the spheroid (Gebhardt et al. 2000, Ferrarese \& Merritt 2000).

On a similar timescale, our understanding of the highredshift Universe changed dramatically with the advent of the SCUBA array at the James Clerk Maxwell Telescope. It appears that a significant (perhaps dominant) fraction of the star-formation in the high-redshift Universe $(z>1)$ took place in highly dust-enshrouded galaxies (Smail et al. 1997, Hughes et al. 1998, Barger et al. 1998, Eales et al. 1999). These exceptionally luminous systems are the analogues of local Ultra-Luminous Infrared Galaxies (ULIRGs). The major difference is that SCUBA sources appear to dominate the high-z cosmic energy budget, while locally ULIRGS are rare and unusual events. The discovery of the SCUBA populations was heralded by many as the discovery of the major epoch of dust-enshrouded spheroid formation (Lilly et al. 1999, Dunlop 2001, Granato et al. 2001).

Given the strong link suspected between the formation of the black hole and its host spheroid, one might expect the most massive newly forming galaxies (i.e. the SCUBA sources) to contain powerful accreting black holes. The first Chandra observations of SCUBA sources, however, find that only a very small fraction $(\sim 5 \%)$ contain a detectable quasar

Correspondence to: omar@ roe.ac.uk
(Fabian et al. 2000; Hornschemeier et al. 2000, Severgnini et al. 2000, Almaini et al. 2002; see Figure 1). The implication is that the major episode of star-formation does not coincide with the epoch of visible quasar activity.

In this paper I review the implications of these results and discuss the possibility that sub-mm sources and quasars represent different stages in an evolutionary sequence.

\section{The potential role of the quasar in galaxy formation}

A number of authors have proposed models to explain the tight black-hole/bulge mass relationship (Silk \& Rees 1998, Fabian 1999, Archibald et al. 2002). Although the details differ, the critical idea is the accreting black hole grows in parallel with the bulge until some process (possibly quasar winds) expel the gas and terminate the star formation.

The potential importance of the black hole can be readily illustrated when one considers the total energy generated by a quasar over its lifetime. If we assume growth by accretion to a total mass $M$ with the usual efficiency $\mu(\sim 0.1)$, folding in the typical black-hole/bulge mass ratio of $\sim 0.1 \%$, the quasar energy is therefore $0.001 \mu M_{\text {bulge }} c^{2}$. If we compare this to the typical binding energy of a massive bulge, $\frac{1}{2} M_{\text {bulge }} \sigma^{2}$ (where $\sigma \sim 300 \mathrm{~km} \mathrm{~s}^{-1}$ ), we find that less than $1 \%$ of the energy generated by accretion would be sufficient to completely destroy the host (Fabian, Wilman \& Crawford 2002). The key issue is to determine what fraction of the quasar energy is transferred to the host galaxy (e.g. by winds) compared to that which escapes. 
A controversial possibility, therefore, is that quasars may have played a critical role in the process of galaxy formation itself. A fundamental feature of these models, however, is that the bulk of the growth of the black hole is coeval with the formation of the spheroid. Why, then, are only $\sim 5 \%$ of SCUBA sources detected in X-rays? There are at least three possible explanations, two of which are evolutionary in nature while the other is based on a duty cycle of AGN feeding:

\section{(i) The duty cycle model}

In this model, the majority of the black holes within the SCUBA sources are simply not being fueled. With starformation rates of $\sim 1000 M_{\odot} \mathrm{yr}^{-1}$ (as required to power the most luminous SCUBA sources) the resulting stellar winds and supernovae should certainly generate a plentiful supply of turbulent gas, but transfering this material to the sub-parsec scale of an accretion disk is by no means guaranteed (Shlosman, Begelman \& Frank 1990). A stopstart duty cycle of fueling could therefore explain the lack of an observable quasar at an any given epoch. This would, however, be in stark contrast to the local counterparts of SCUBA sources (the luminous low-z ULIRGs) where at least 50 per cent show some evidence evidence for an active nucleus (Sanders \& Mirabel 1996).

(ii) Evolutionary model A: Growing black holes

Archibald et al. (2002) have proposed a model in which the central quasar is alive but still growing. This is based on the hypothesis that a black hole is likely to grow from a small seed $\left(\sim 100 M_{\odot}\right)$ and hence, even accreting at the Eddington limit, it will require $\sim 5 \times 10^{8}$ years to reach a sufficient size to power a quasar. At this stage the peak star-formation activity may have ended, leading to a natural lag between the SCUBA phase and the subsequent luminous quasar. This "growing quasar" model could explain the weak levels of X-ray emission detected in many SCUBA sources by Alexander et al. (2002) in the 2Ms observations of the Hubble Deep Field North.

(iii) Evolutionary model B: The Compton-thick AGN

Perhaps the most popular explanation is that the SCUBA sources contain accreting quasars, but these are obscured by Compton thick material $\left(N_{H} \gg 10^{24}\right.$ atom $\mathrm{cm}^{-2}$ ) which is later blown away as the black hole grows (e.g. Fabian 1999). It should be noted, however, that a Compton-thick explanation would require almost isotropic obscuration, rather than a standard "Unified Scheme" torus, since only $\sim 5 \%$ of SCUBA sources are detected by Chandra. This would be consistent with models in which a nuclear starburst both fuels and obscures the active nucleus (Fabian et al. 1998). Perhaps 50 per cent of all local AGN are Compton-thick (Maiolino et al. 1998), but the abundance of such AGN at high redshift (and high luminosities) is currently unclear. It is conceivable that future constraints on the local space density of supermassive black holes may place tighter constraints on the prevalence of Compton-thick accretion in the Universe, particularly once the contribution of "Comptonthin" black hole growth can be ascertained more accurately from the Cosmic X-ray background.

\section{Additional observational constraints}

So far we have discussed the tight local bulge/black-hole relationship and the observation that the majority of high-z SCUBA sources (which are arguably proto-spheroids) are not detected as luminous X-ray sources with Chandra. Below I outline a number of other recent observational constraints on the links between sub-mm sources and quasars.

\subsection{Sub-mm observations of powerful high-z quasars}

Another route to exploring the link between black-hole and spheroid formation is to study the star-formation history of the hosts of known active black holes. Archibald et al. (2001) performed this experiment for the most massive black holes by undertaking a submillimetre study of a sample of $\simeq 50$ radio galaxies spanning a wide range in redshift $(1<z<$ $5)$. The principal result of this study was that the sub-mm detectability of powerful radio galaxies was found to be a strong function of redshift, with a detection rate of $\simeq 75 \%$ at $z>2.5$ compared to only $\simeq 15 \%$ at $z<2.5$. The average sub-mm luminosity of the radio galaxies in the sample was modeled and found to increase with redshift, with $L_{850 \mu m} \propto$ $(1+z)^{3}$, even at $z>3$.

Attempting a similar analysis with X-ray selected AGN, Page et al. (2002) recently observed a sample of 8 absorbed AGN over a wide redshift range but with similar intrinsic power. Although the sample is small, it is intriguing that only the 4 AGN with highest redshift $(z>1.5)$ were detected by SCUBA, with luminosities an order of magnitude higher than one would expect from AGN heating.

Combined, these results provide rather convincing evidence that the hosts of massive black holes at high redshift are very different to the relatively passive elliptical hosts of low-redshift AGN. Specifically, irrespective of whether the dust is heated primarily by the UV output of young stars or by the AGN itself, it is clear that the mass of dust in highredshift AGN hosts is much greater at $z>2$.

\subsection{Sub-mm observations of typical Chandra sources}

The obvious extension of the sub-mm programmes described above is to target more complete, flux-limited samples of AGN. It is unclear, for example, whether radio-loud quasars have sub-mm properties which are representative of AGN as a whole.

As a first step, we have performed a sub-mm stacking analysis at the positions of Chandra sources in the ELAIS $\mathrm{N} 2$ field. We find a mean flux of $1.25 \pm 0.4 \mathrm{mJy}$ (Almaini et al. 2002), consistent with the similar work of Barger et al. (2001). Redshifts are accumulating, but we find intriguing evidence that the stacked sub-mm flux is a strong function of optical faintness (Figure 2). This may reflect enhanced dust emission from obscured and/or high-z AGN, although this could be strongly influenced by the negative K-correction which operates in the sub-mm regime. Soon we will obtain redshifts for most of these AGN and hence be able to study their mean sub-mm properties as a function of both luminosity, cosmic epoch and absorbing column. 


\subsection{The clustering of Chandra and SCUBA sources}

Despite the low coincidence rate, there is growing evidence that bright SCUBA sources are strongly clustered with field AGN (Almaini et al. 2002; see Figure 1), a result which has now been confirmed in two further independent fields (Almaini et al. 2003, in preparation).

At first sight this would suggest that these populations, though distinct, are tracing the same large-scale-structure and are therefore coeval. This is puzzling, however, given that the best constraints on the SCUBA sources suggest that the majority lie at $z>2$ (Ivison et al. 2002, Smail et al. 2002), while the results of X-ray surveys (e.g. Hornschemeier et al. 2001) suggest $\bar{z} \sim 1$.

I propose an alternative explanation which invokes gravitational lensing, motivated by the particularly steep sub-mm source counts. In the 'weak' lensing regime, for example, where the typical magnification $\mu$ is not significantly greater than unity, one can readily demonstrate that a population with cumulative number counts given by a power law of index $\beta$ will be modified as follows:

$N^{\prime}(>S)=\mu^{\beta-1} N(>S)$

Observed sub-mm number counts have a slope with $\beta \simeq$ 2.5 (possibly steepening beyond $8 \mathrm{mJy}$ ). Foreground large scale structure (e.g. foreground groups) can readily lead to a magnification of $\mu \simeq 1.2-1.3$. Thus in the vicinity of such structure one would expect an enhancement in number counts of $30-50 \%$ which could easily produce a positive cross-correlation with foreground populations (as traced by the Chandra sources).

In conclusion, it seems likely that the appearance of largescale structure may simply be an artifact of gravitational lensing on a flux-limited sub-mm survey, giving the misleading impression that SCUBA galaxies and Chandra sources are coeval.

\section{Conclusions}

A number of independent lines of evidence now suggest that sub-mm sources and high redshift quasars represent different stages in the evolution of a massive proto-spheroid. First of all, the bright SCUBA population (arguably the progenitors of local massive elliptical galaxies; Lilly et al. 1999) are, in general, not detected by Chandra, despite the likelihood that these host (or will develop) black holes in excess of $10^{9} M_{\odot}$. This strongly argues for two distinct stages of evolution, although it is unclear whether the central black hole is dormant, still growing or obscured by Compton-thick material.

Additional evidence comes from targeted sub-mm observations of powerful radio galaxies, which in principle ought to be sign-posts of massive accreting black holes irrespective of the obscuring column. The sub-mm luminosity appears to evolve very strongly with redshift. Similar (though more tentative) results have also emerged from sub-mm observations of X-ray selected AGN.

Finally I note that the apparent clustering observed between SCUBA sources and Chandra-selected AGN, which

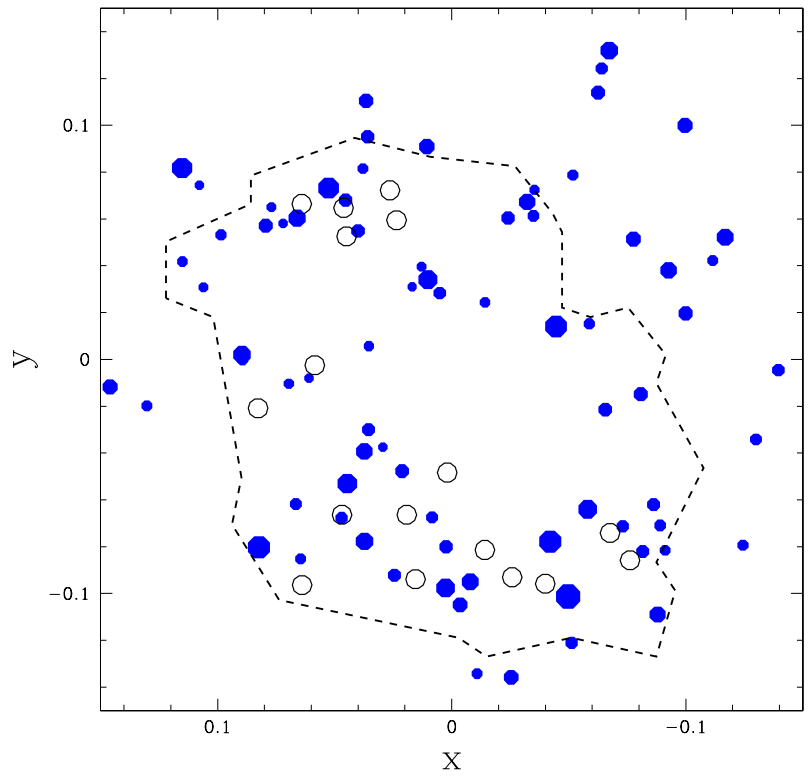

Fig. 1. Illustrating the distribution of Chandra sources (filled points) and SCUBA sources (open circles) in the ELAIS N2 field. The size of the X-ray points are proportional to the $\log$ of their flux. The dashed regions show the extent of the SCUBA coverage. While the overlap between these populations is small $(1 / 17)$ the two populations appear to trace the same large-scale structure (see Almaini et al. 2002).

at first sight suggests that these populations are coeval, may actually be an artifact caused by the effects of gravitational lensing.

Acknowledgements. OA acknowledges the considerable support provided by the Royal Society.

\section{References}

Alexander, D.M., et al.: 2002, ApJ, in press

Almaini, O., et al.: 2002, MNRAS, in press, (astro-ph/0108400).

Archibald, E.N. et al.: 2001, MNRAS, 323, 417

Archibald, E.N. et al.: 2002, MNRAS, 336, 353

Barger, A., et al.: 1998, Nat, 394, 248

Barger, A., et al.: 2001, ApJ, 560, L23

Dunlop, J.S., 2001: in proceedings of UMass/INAOE conference

Eales, S., et al.: 1999, ApJ, 515, 518

Fabian, A.C., Barcons X., Almaini O., Iwasawa K.: 1998, MNRAS, 297, L11

Fabian, A.C.: 1999, MNRAS, 308, L39

Fabian, A.C., et al.: 2000, MNRAS, 315, L8

Fabian, A.C., Wilman, R.J., Crawford, C.S.: 2002, MNRAS, 329, L18

Ferrarese, L., Merritt D.: 2000, ApJ, 539, 9

Gebhardt, K., et al.: 2000, ApJ, 539, 13

Gendreau, K., et al.: 1995, PASJ, 47, L5

Granato, G.L., et al.: 2001, MNRAS, 324, 757

Hornschemeier, A.E., et al.: 2000, ApJ, 541, 49

Hornschemeier, A.E., et al.: 2001, ApJ, 554, 742

Hughes, D.H., et al.: 1998, Nat, 394, 241

Ivison, R.J., et al.: 2002, MNRAS, 337, 1

Kormendy, J., Richstone, D.: 1995, ARA\&A 33, 581

Lilly, S.J. et al. 1999, ApJ, 518, 641 


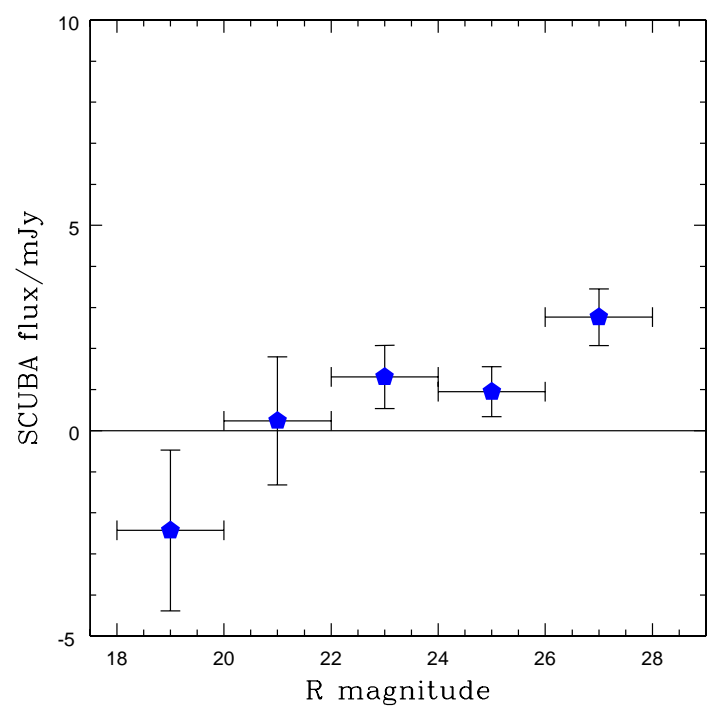

Fig. 2. Stacked sub-mm flux as a function of optical magnitude for the 55 Chandra sources lying within the SCUBA map for the ELAIS N2 field. The tentative conclusion is that obscured and/or high-z Chandra sources may be more luminous in the sub-mm (Almaini et al. 2003, in preparation).

Magorrian, J., et al.: 1998, AJ, 115, 2285

Maiolino, R., et al.:1998, A\&A, 338, 781

Page, M.J. et al.: Science, 294, 2516

Sanders, D.B., Mirabel I.F.: 1996, ARA\&A, 34, 749

Scott, S.E., et al.: 2002, MNRAS, 331, 817

Setti, G., Woltjer L.: 1989, A\&A, 224, L21

Severgnini, et al.: 2000, A\&A, 360, 457

Shlosman, I., Begelman, M.C., Frank, J.: 1990, Nature, 345, 679

Smail, I., Ivison R.J., Blain A.W.: 1997, ApJ, 490, L5.

Smail, I., Ivison R.J., Blain A.W., Kneib J.P.: 2002, MNRAS, 331, 495 